\title{
A New World Geographic Reference System
}

\author{
Keith C. Clarke, Peter H. Dana, \\ and Jordan T. Hastings
}

\begin{abstract}
A new global georeferencing system—-the World Geographic Reference System (WGRS)—is proposed. This system has particular advantages for location description and communication with electronic devices, i.e., in digital environments that are shared between humans and machines. The new World Geographic Reference System strikes a compromise between the dominant use of numbers in established scientific coordinate systems, such as latitude/longitude, and the colloquial preference for names, particularly names of administrative units and populated places, in everyday life. Specifically, WGRS defines a system of uniform regional grids, each 100x100 km in extent, anchored on and named by prominent cultural and/or physical features. Subsets of these regional grids, called local grids, which are particularly adapted to smaller places, also may be defined. A location within a regional or local grid is georeferenced by suffixing the grid identifier with a coordinate string of dotted-digit-pairs that represent interleaved Cartesian x-y displacements from the grid origin. A typical WGRS locator, for example, is US.DC.WAS.54.18.28, representing a 100x100 m area, the southwest corner of which is $\mathbf{0 . 5 1 2}$ of the way across (east) and $\mathbf{0 . 4 8 8}$ of the way up (north) in the Washington, D.C., grid, roughly the lawn surrounding the Washington Monument. This locator, which is easily interpreted by both humans and machines, also may be effectively communicated between them via computer networks using a notation, such as "wgrp://US.DC.WAS.54.18.28." The similarity of WGRS locators (WGLs) to Uniform Resource Locators (URLs) on the Internet is intentional, facilitating their use in Web and wireless application interfaces, especially those employed in location-based service systems.
\end{abstract}

KEYWORDS: Coordinate systems, geocoding, georeferencing, mapping grids

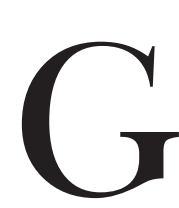

\section{Introduction}

eospatial technologies are ultimately dependent upon the georeferencing grid systems used to assign coordinates to locations on or near the surface of the Earth. Cartography and geodesy have devised a multitude of such grid systems over time for various purposes, including the Universal Transverse Mercator (UTM) system, the U.S. Military Grid Reference System (MGRS), the State Plane Coordinate System (SPCS), the British National Grid (BNG), and many others. Regardless of their exact technical specifications, all such grid systems share: 1) a structured terminology and explicit syntax for coordinate references; 2) a specific mechanism for producing coordinates in relation to a geodetic datum and projection system;

Keith C. Clarke is professor and chair at the Department of Geography, University of California Santa Barbara, Santa Barbara, CA 93106 U.S.A. E-mail: <kclarke@geog.ucsb.edu>. Peter H. Dana is a research fellow and lecturer at the Department of Geography, University of Texas at Austin, Austin, TX 78712, U.S.A. E-mail: < pdana@mail.utexas.edu>. Jordan T. Hastings is a doctoral student at the Department of Geography, University of California Santa Barbara, Santa Barbara, CA 93106, U.S.A. Email: <jordan@geog.ucsb.edu>. and 3) a unique and exhaustive system of spatial indexing to either point or areal locations. Spatial indexing permits tiling of grids according to a given pattern over the region of interest. Tiling approaches have varied from 6-degree longitudinal strips (UTM), to 6 by 8 degree blocks (MGRS), to the boundaries of states or counties within states (SPCS). Surprisingly, little cartographic research has focused on either identifying the benefits of particular grid systems or comparing them analytically. Goodchild (1994) specified a set of criteria for grids, including accuracy, authority, definitiveness, exhaustiveness, hierarchy, simplicity, succinctness, tractability, uniqueness and universality. Some of these criteria were analyzed further by Kimerling et al. (1999) and by Clarke (2002). In designing the present World Geographic Reference System (WGRS), we have emphasized simplicity above most of the other criteria, especially exhaustiveness and uniqueness.

Given a grid system, precise location references are made by numeric offsets (e.g., eastings and northings) within its tiles. The complete georeference is typically encoded in a single alphanumeric string, or geocode, that gives both the identifier (or index) of the base grid and the offsets within it in some form. The length of the geocode determines the precision of the system. In UTM, for example, the top of the 
dome of the U.S. Capitol in Washington occurs at “325755m E, 4306467m N; Zone18 N (NAD83)." Here the leading numbers define the easting and northing coordinates, indexed to zone 18 (North), and NAD83 specifies the datum. ${ }^{1}$ Alternative recent systems have varied both the nature of the tiling and the syntax of geocodes (Tobler and Chen 1986; Dutton 1997).

The primary benefits of encoded georeferences are simplicity and universality, while some geocodes also provide explicit indication of precision. Automated systems can take advantage of compression schemes when many geocodes are stored together (Clarke 1995). However, geocodes tend to be cryptic and so are susceptible to being misstated or confused. Problems also arise with grid tile overlap and projection distortion, particularly near grid edges and at the poles. The inevitability that important features will fall on grid seams has been termed the "second law of geography" (Clarke 1995), i.e., that things of highest geographic interest lie at the intersection of several unfortunately tiled maps or images. Overall, georeferencing grid systems may be mathematically succinct, but they are seldom user-friendly. The BNG, devised and promulgated for a relatively small region, is a notable exception and widely used.

By contrast to these formal, mathematical approaches, the majority of georeferencing in everyday life is accomplished in relation to named places, i.e., well known cultural and/or physical geographic features (Dana and Hancock 1997). Place names also appear prominently in street addressing and routing instructions, and in many legal descriptions. Postal codes are a conspicuous exception (Raper et al. 1992) to the human preference for names over numbers and codes. Thus, while the UTM geocode given above locates the Capitol dome within one meter, so does the simple text string "Top of U.S. Capitol, Washington, D.C., USA," which also uses 40 characters (including punctuation and spaces). The former notation requires a map expert to decode, while the latter is accessible to anyone with knowledge of basic geography, presuming the place name has a generally accepted meaning. Automated conversion of place names to geodetic coordinates is error-prone, however, and often imprecise; metrics between named places are necessarily approximate. We propose WGRS as a compromise between the two georeferencing systems, mathematical and conventional, preserving the essential benefits of each.

The intent of WGRS is to create a firm foundation for location description and communication with electronic devices in the 21 st century. We begin from the position that the geographical reference systems in common use (place names, cultural landmarks, street addresses) are undeniably more user-friendly than grid coordinates of any kind. In these systems, point locations are typically given via street addresses and/or intersections, while more general locations are identified by the names of prominent places. The latter are often nested, e.g., "the church in Central Park," since human geographic behavior is across-scale and multi-scale in nature (Goodchild and Proctor 1997). Following these precepts, WGRS is both colloquial and hierarchical, intermixing mnemonic and numeric styles of georeferencing in a notation equally accessible to people and machines. The WGRS locator for the U.S. Capitol dome, again with one-meter precision as given earlier, is the 24-character code US.DC.WAS.54.38.58.56.29. This geocode is compact and succinct, facilitating its use in print and speech as well as electronic communications that involve global positioning systems (GPS), geographic information systems (GIS), and the wide array of emerging location-aware appliances such as cell-phones, personal digital assistants, and in-car navigation systems. In both written and verbal forms, WGRS is specifically designed for ease of use by people and machines, to facilitate their interaction on everyday geographic chores. The WGRS notation also emphasizes locality in georeferencing, thereby encouraging its adoption and preserving geographic heritage. This localization has the additional benefit that spatial error is minimized in practical terms, not over an arbitrary map region, but specifically centered on places of maximum interest and use.

The World Geographic Reference System is the outcome of collaboration between a privatesector location-based services (LBS) provider, UDS Directory Corp., d/b/a go2 Directory Systems (Go2; http://www.go2.com), and the National Center for Geographic Information and Analysis (NCGIA; http: //www.ncgia.org) at the University of California Santa Barbara. Various features of WGRS are covered by U.S. patents (Hancock and Dana 1998, et seq.). Go2 intends to make a significant portion of the WGRS technology available to software developers, other LBS providers and academic researchers on a community source-license basis (Rosenberg 2000). To further encourage its use, WGRS may be proposed for incorporation in Internet and Web specifications developed through the Internet Engineering Task Force (http://www.ietf.org/) and the World Wide Web Consortium (http://www.w3.org/). Essential components of WGRS, including its technical principles and computer algorithms and databases, will be posted and maintained on the Web and also published periodically in hardcopy forms. The Go2 Web site

${ }^{1}$ The bare minimum UTM encoding is 15 digits,"325755430646718", plus 1 (bit) more for the hemisphere. 


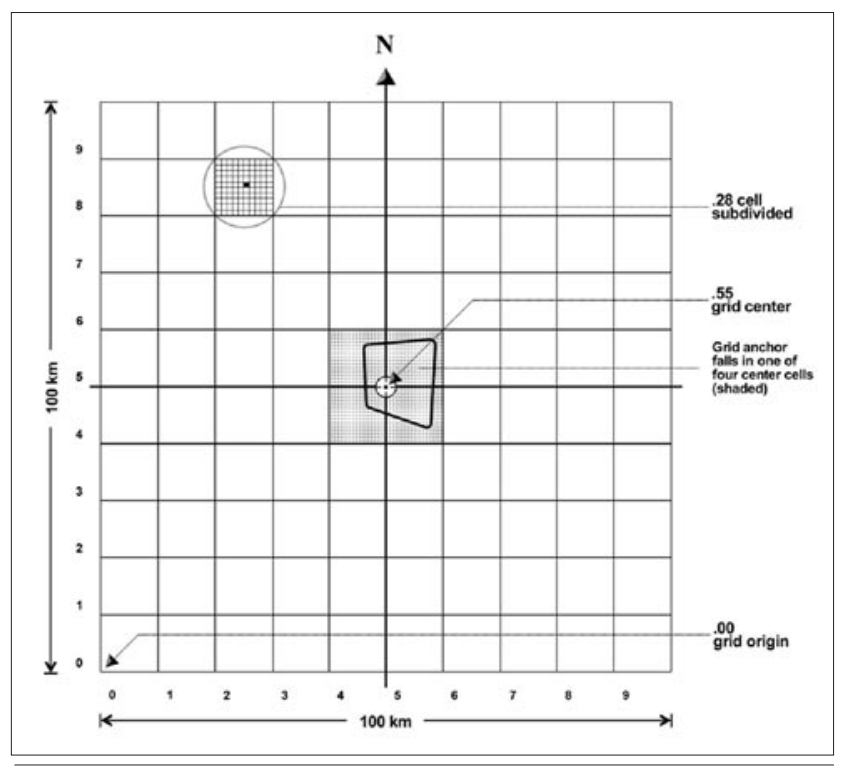

Figure 1. A single WGRS grid.

provides software (http://www.go2online.com/webbrowser/ indexGo2AddressWizard.cfm) that allows conversion of zip codes and street addresses into WGRS locators.

This paper introduces the World Geographic Reference System conceptually, formally and independently of its applications, since WGRS stands alone as a unique spatial indexing system. We conclude with a discussion of WGRS's anticipated advantages and challenges.

\section{Grid Definition}

The geometric basis for WGRS is the regional grid (RG): a 100x100 km Cartesian mapping framework, comprising one hundred $10 \times 10 \mathrm{~km}$ cells, each of which in turn comprises one hundred $1 \times 1$ $\mathrm{km}$ sub-cells, etc. (Figure 1). Using a stereographic projection, such a grid may be readily constructed anywhere on Earth; different computational methods apply to polar, equatorial, or oblique cases (Snyder 1987). WGS-84 is taken as the geodetic datum. ${ }^{2}$ The stereographic projection is chosen despite the fact that it is not equal area, because it is conformal with error increasing uniformly outward from the central point of projection, thus minimizing error at the point to which it is applied. Alternative projections are possible.

The center-point for the grid, which is also the center of the projection, is placed at the lower left corner of the grid's .55 cell, i.e., the intersection of the grid center-lines. The grid origin is shifted to the lower-left corner of its $\mathbf{. 0 0}$ cell by a false easting and a false northing of 50,000 m each (Figure 1). The grid is oriented such that its vertical center-line runs due north-south, coincident with the local meridian at the center-point.

Locations within a grid, which are always square areas, are notated by a string of dotted-digit-pairs (DDP), which are interpreted hierarchically. Within each pair, the first digit represents the lower-left x-coordinate (easting) and the second digit represents the lower-left y-coordinate (northing) in the hierarchy, which applies initially to the $100 \times 100$ grid square. Each digit-pair subdivides its antecedent grid space decadally in tiers, i.e., the first pair resolves to a cell, the second pair to a sub-cell, etc. Thus, for example, the location .55 denotes the upper-right cell (10) 10 $\mathrm{km}$ area) of the four central cells of any grid; whereas .28 .55 denotes a sub-cell $(1 \times 1 \mathrm{~km}$ area) within the grid's .28 cell (see Figure 1).

The mnemonic basis for WGRS is the place name (PN): the official, fully spelled-out name of a cultural or physical geographic feature on the Earth. In general, PNs are as specified in the National Imagery and Mapping Agency (NIMA) world gazetteer of place names, ${ }^{3}$ which also includes their locations in MGRS notation. Place names are preferably anglicized using the International Standards Organization (ISO) 8859 Extended Latin Character Set (Latin 1), although multilingual versions of names can be accommodated. For convenience in compositing strings of names (below), a PN generally will be shortened to a place name abbreviation code (PNAC), which is any accepted abbreviation for the place that is unambiguous within the context of that string. In the U.S., the Federal Information Processing Standard (FIPS) state and county name codes are adopted (see http://www.itl.nist.gov/fipspubs/by-num.htm/, documents 5-2 and 6-4); analogous conventions and/or standards apply in many countries.

Place names and/or place name abbreviation codes are composited hierarchically into place name strings (PNS), using dot separators similar to the DDPs in grid locations. The composition progresses from smaller to larger spatial scales, left to right. The coarsest (left-most) element in the PNS is the ISO3166 two-character world country code, e.g., 'Us' for the United States; the intermediate elements are country-dependent codes for administrative units-states, provinces, regions, and departments; the finest (right-most) element is typically a code for a populated place. For example, Washington, D.C., might be WGRS-coded as US.DC.WAS.

\footnotetext{
2 Specific WGS-84 parameters will be selected to establish a consistent one-to-one mapping of geodetic coordinates to WGRS, and vice-versa, on all computing platforms.

3 NIMA maintains a gazetteer server with access to 3.5 million such features in a database, at http://www.nima.mil/gns/html/.
} 
Each regional grid is identified by a place name string; dotted-digit-pair suffixes further refine locations within them. Thus US.DC.WAS.55, US.DG.WAS.55.55, and US.DC.WAS.55.55.55 represent progressively tighter focus on the center of Washington, while US.DC.WAS.34.98 refers to a $1 \times 1 \mathrm{~km}$ area, the southwest corner of which lies $11 \mathrm{~km}$ west and $2 \mathrm{~km}$ south of the city center. Upper tiers at the left of the location string can be suppressed if the context is evident; similarly, lower tiers can be truncated on the right when the desired spatial resolution is achieved. Well formed, i.e., syntactically and semantically correct, unambiguous WGRS georeferences are termed World Geographic Locators (WGL). The notation is intentionally similar to that of Uniform Resource Locators (URL) (World Wide Web Consortium 2002) on the Web.

The unique value of WGRS is that its underlying framework, that of regional grids, is predominantly culturally defined: regional grids are generally selected to coincide with the most populous places. The centerpoint of each regional grid is derived by rounding the listed geographic coordinates, or anchor point, ${ }^{4}$ for the defining real-world place to the nearest 0.05 degrees (3 minutes) of latitude and longitude, typically $<5$ $\mathrm{km}$. By this definition, the "center" of a place, e.g., its business district or cultural downtown, will always occur in or among the four central cells $(. \mathbf{4 4}, .45, .54$, and .55) of its regional grid, and the cross $(+)$ where these cells meet defines the four cardinal directions at that point on the grid (refer to Figure 1).

As a comprehensive example, the WGRS grid for Washington, D.C., US.DC.WAS, has the anchor point $\left(38^{\circ} 53^{\prime} 42^{\prime \prime} \mathrm{N}, 77^{\circ} 02^{\prime} 12^{\prime \prime} \mathrm{W}\right)$, at the Zero Milestone marker (http://www.fhwa.dot.gov/infrastructure/zero.htm). The corresponding RG center-point is $\left(38.90^{\circ} \mathrm{N}, 77.05^{\circ} \mathrm{W}\right)$, approximately $1 \mathrm{~km}$ northwest of the Mall (Figure 2). The Washington Monument ${ }^{5}$ is one of many well known landmarks in this grid. The following World Geographic Locators apply:

- US.DC.WAS - the full $100 \times 100 \mathrm{~km}$ area (grid) centered on the Zero Milestone marker, i.e., extending $50 \mathrm{~km}$ $\mathrm{N}, \mathrm{E}, \mathrm{S}$, and $\mathrm{W}$ from the center-point;

- WAS.55 - the 10x10 km area (cell) immediately NE of the grid center; the prefix tiers US.DC. have been dropped, optionally;

- WAS.54 - the 10x10 km area (cell) immediately SE of the grid center;

- WAS.54.18 - the $1 \times 1 \mathrm{~km}$ area (sub-cell) beginning 1 $\mathrm{km}$ across and $8 \mathrm{~km}$ up within the $\mathbf{. 5 4}$ cell;

- WAS.54.18.28 - the 100x100 $\mathrm{m}$ lawn area surrounding the Washington Monument;

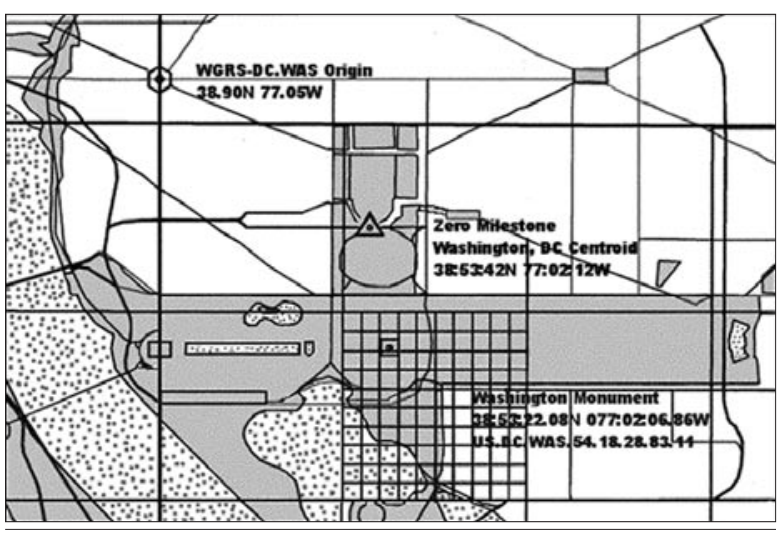

Figure 2. Geographic and WGRS references for the Washington Monument.

- WAS.54.18.28.83 - the 10x10 m square approximating the concrete base of the Washington Monument itself; and

- US.DC.WAS.54.18.28.83.11-full WGL for the $1 \times 1$ $\mathrm{m}$ center area directly beneath the apex of the Washington Monument.

In relation to a given regional grid (RG), any number of local grids (LGs) may be specified, each of which exists on the coordinate framework defined by that RG (Figure 3). The defining RG is referred to as the parent grid and the LGs as its children, all of which are siblings. Local grids also are identified by place names, which are syntactically indistinguishable from those of regional grids. Hence, the World Geographic Locator (WGL) geocodes based on local and regional grids may be used interchangeably. Also by this construction, the sub-cell (second DDP) and all lower tiers of an LG-based WGL are identical to those of the parent RG-based WGL for the same place, avoiding proliferation of only slightly different gridding frameworks.

Typically, local grids are subsets of the defining regional grid's cells; however, limited supersets (groupings) are also possible (refer to Figure 3). For consistency, both types of grids designate $\mathbf{. 5 5}$ as their central cell. To accomplish this, the LG is shifted, if necessary, by an integral number of cells east (or west) and north (or south) in relation to its parent RG. In general, only a few cells of a local grid, roughly corresponding to the perimeter of the locality, are treated as valid or activated; the balance are un-activated, or masked. In this way, the same point can have both a local reference and a broader regional reference, depending on the user's

\footnotetext{
4 Typically, but not necessarily its centroid; definition of centroids is beyond the scope of this discussion.

5 Listed by the National Geodetic Survey as Washington Monument 1913 PID=HV4442 NAD 83 (1993)-38 5322.08377 (N) 07702 06.86378 (W). This location is described in the data sheets as "the center of the aluminum tip that surrounds the monument. This tip has apparently been burned by lightning, as the top is about $1 / 2$ inch square (1934)." In 2000 , the monument was reworked and the tip was replaced.
} 


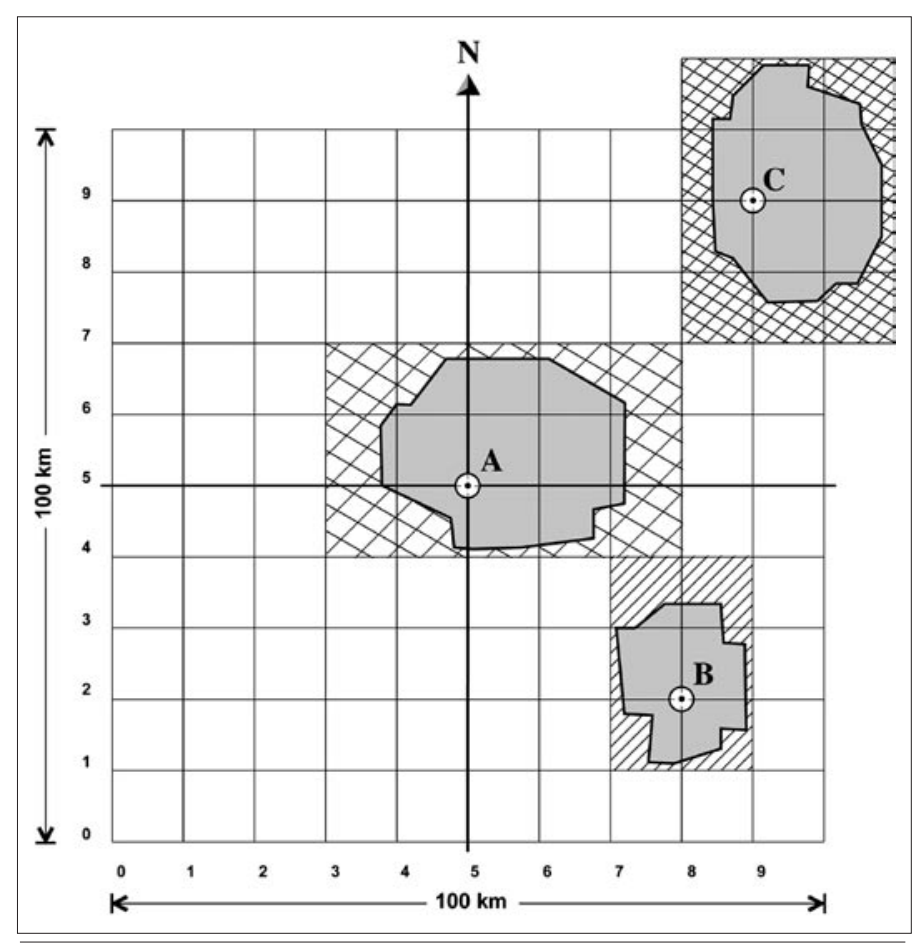

Figure 3. Shifting and masking of local vs. regional WGRS grids. Anchor $A$ defines a regional grid; $B$ and $C$ are sibling local grids within it. Place perimeters are shown with activated grid extents cross-hatched.

application, although only one local grid can apply (be active) at any given time. The parent RG is always considered activated over its full geographic extent. The activated extent of a LG may fall outside the perimeter of its parent RG, however, in border-cell cases. For example, in Figure 3, the LG cell C.55 is synonymous with its parent RG cell A.99, and hence 6 cells of $\mathbf{C}$ fall outside $\mathbf{A}$ altogether. The maximum effective reach of a regional grid is thus $190 \times 190 \mathrm{~km}$, in the (unlikely) event that multiple large local grids appear along all four of its borders.

Regional grids are designed to overlap to a limited extent to ensure complete coverage of a given geographic territory; their dependent local grids also may overlap, in populous regions substantially, both with themselves and other regional grids. This over-tiling, which is unusual and avoided in other georeferencing frameworks, does not cause problems for WGRS because each place is referenced to a single autonomous grid, either a regional grid or a local grid. Where a candidate place for a local grid falls in two or more regional grids, it is activated in only one of them.

Local grids serve to co-relate groups of places that occur within the ambit of a regional grid, for example, the continuum of populated places that often appears in a large metropolitan area. The localities of Alexandria, VA, and Bethesda, MD, are identifiable sibling LGs of the Washington, D.C., (WAS) regional grid. Also, Washington National Airport (DCA) is a single-cell local grid, defined as coincident with the WAS.44 cell. Then US.DC.DCA.55.12.34 and US.DC.WAS.44.12.34 are equivalent WGLs, or numeric aliases, for the same spatial location. Aliases accommodate local conventions and preferences. The administrators of DCA may view the airport's service area radially from that center, while airline passengers may relate to the larger place, Washington, D.C. Residents of Alexandria, VA, and Bethesda, MD, are free to identify with those places in preference to either the national capital or its downtown airport. Viewed from afar, these distinctions are often immaterial: foreign tourists likely will consider and georeference all four places together within the single ambit of the wAS regional grid.

\section{Grid Selection}

The WGRS grids are uniformly 100x100 km in extent. In populous regions, the anchor points for a set of grids should be chosen carefully in order to minimize the regional over-tiling of grids, when viewed at the state level. In sparsely settled areas, the opposite circumstance arises: the set of well-known named cultural and physical features may be insufficient to define a covering set of grids, in which case arbitrary benchmarks may be chosen for anchors as convenient. On the open ocean, and elsewhere if desired, a graticular mapping framework based on fixed increments of latitude and longitude also may be used to define systematic anchors for a regular quilt of grids.

Given the global nature of WGRS, and the plethora of place names globally, an automated method for selecting both regional and local grids and assigning place name abbreviation codes to them is required. One such method, demonstrated to be workable for the United States, follows. First, a list of the largest populated places within the territory of interest (here California, i.e., US.CA) is extracted from the GNIS database. ${ }^{6}$ This database contains over 168,100 populated places with coordinates in the U.S.; however, only about 22,100 carry current (1990 or later) population estimates. From these, duplicate and variant names (e.g., City of Los Angeles vs. Los Angeles; San Buenaventura vs. Ventura) are removed, leaving about 21,750 places, which are then sorted by state

6 The Geographic Names Information System (GNIS) is maintained by the U.S. Geological Survey for the Board on Geographic Names, at http://mapping.usgs.gov/www/gnis/. 
and population to generate a prioritized list of regional grid candidates. These candidates are thinned in an iterative algorithm that repeatedly: 1) selects, as a regional grid, the largest remaining place, based on population (or other priority status); then 2) removes from the list both this place and all other places that fall within some tolerance of the newly-selected grid perimeter. The algorithm runs until the list is exhausted. As the tolerance is increased, both the number of grids generated and their overlap increase.

California, having an area of approximately 406,000 square kilometers, would require a minimum of 41 regional grids with zero overlap, if that were possible. In fact, the top 38 grids for California selected by the above algorithm, with $20 \mathrm{~km}$ overlap (i.e., excluding candidates within a central $60 \times 60 \mathrm{~km}$ area of each other) covered $\sim 69$ percent of the land area of the state. With 111 grids similarly selected, but only $2 \mathrm{~km}$ overlap, $\sim 88$ percent of the land area was covered. By experimentation, a 5-km overlap was found to be near-optimal, producing a selection of 68 grids with $\sim 86$ percent coverage. The remaining 14 percent of the land area occurred in a mixture of small slivers and some large blocks, particularly in the sparsely settled eastern portion of the State (Figure 4). Using local knowledge of California, 20 more grids were added, and 10 were moved or renamed. Place identity is often a matter of local knowledge (of history, landmarks, etc.) in preference to the formal census and municipal place names derived from gazetteers. The final result for California was a set of 88 regional grids that completely covered the State (Figure 5).

An identical treatment for New York, again using a $5-\mathrm{km}$ overlap, required the addition of only one grid to the 36 regional grids auto-generated by the algorithm. Connecticut was completely tiled into six regional grids by the algorithm, without need for editing.

\section{Discussion}

The World Geographic Reference System is designed to support the convergence of geospatial and telecommunications technologies in consumer devices such as GPS receivers, in-vehicle navigation systems, location-aware cellular telephones, and many expected new products. Such location-based services (LBS) devices necessarily transact spatial references across a human-com-

puter interface via geocodes (NCGIA 2001). To date, geocoding has been largely hidden from public view, although geocodes do appear on GPS receivers and often are printed cryptically along the edges of topographic maps. Increasingly, however, geocodes and digital maps are becoming consumer products, on the Web and in burgeoning wireless devices, notwithstanding the fact that the capabilities of LBS devices and services far exceed the sophistication of most potential users. The rapidly increasing number of interactive maps on the World Wide Web-an obviously dated estimate cites over a million maps a day (Crampton 1998) is forcing public awareness of geographical science, including geocoding.

For all LBS applications, simplicity and clarity of geocodes are critical, both because of the hand-held and mobile devices' limited displays and keyboards, and also because of users' limited attention spans and geographic training. It seems unlikely that the grid systems designed for cartographers, surveyors, and geodesists can easily accommodate the casual user of geospatial technologies, or even should. Locational chores with LBS devices are often approximate, localized, time-sensitive, and typically map-free. Spatial data that come from GPS and GIS, navigational aids, directional signage in the environment, even 


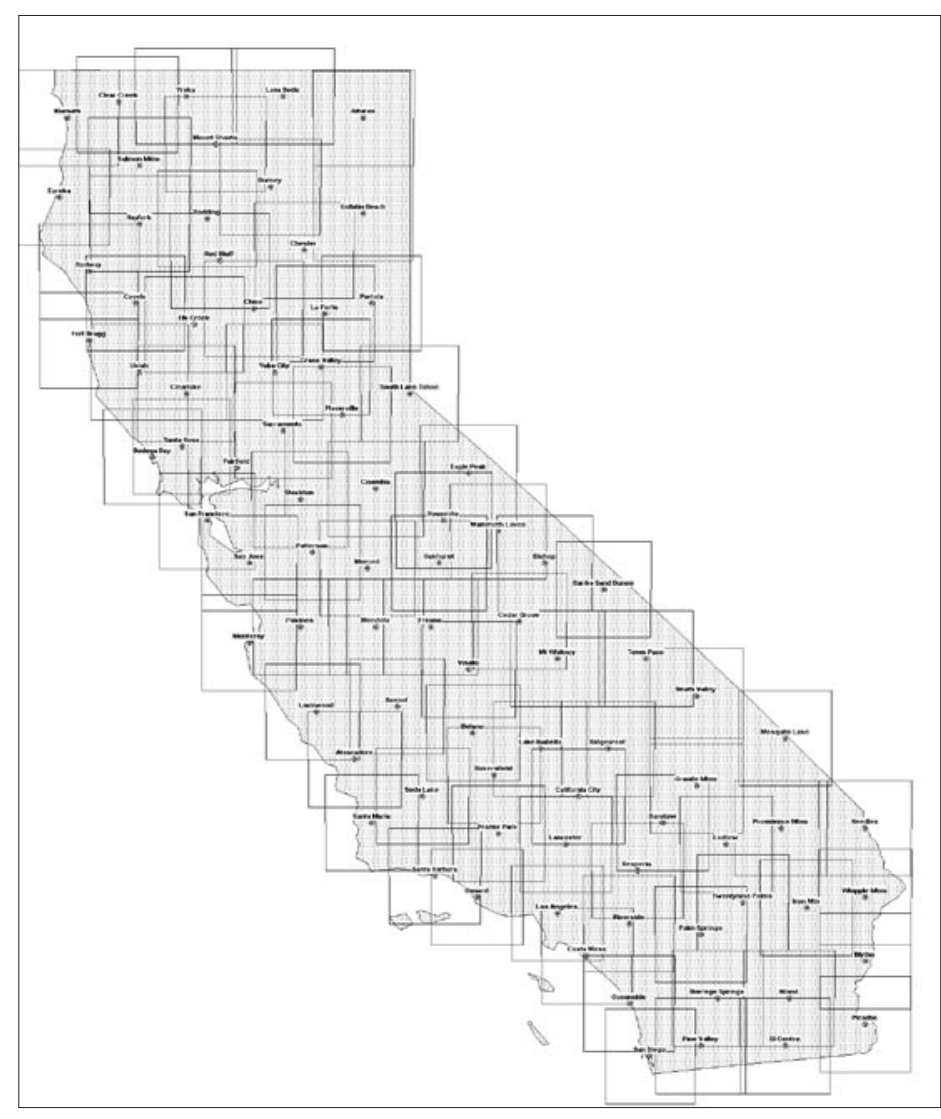

Figure 5. Manually adjusted WGRS grids for US.CA (88 grids, 100 percent state coverage).

maps-all need to be quickly integrated in relation to nearby cultural or physical features. Concise, mnemonic geocodes, such as WGRS provides, can simplify this integration.

For example, a driver has a minor accident in a shopping mall parking lot and calls the local police department to report it. The driver's cellular telephone with on-board GPS could present specific geographic coordinates in one of numerous formats, all of which are error-prone to recite; alternatively, the driver could report the location as "in the parking lot in front of the supermarket at the Fairview Shopping Center," which lacks specificity. By contrast, the WGRS geocode, US.DC.WAS.12.34.56.78 (possibly further shortened to WAS.12.34.56.78), is precise and succinct, with some valuable error checking of both the name string and the digit-pairs built into the syntax. A mangled geocode would be immediately recognizable as such by both humans and machines. If truncated at any point, but still well formed, this geocode would correctly narrow the area of search.

Another point of consumer contact with geospatial data is, of course, the World Wide Web. Curiously, most Web searches are "spaceless:" a search is just as likely to return results from the other side of the country (or the world) as nearby. Yet, if geography makes a difference, then near things should be more related to each other than to those more distant. Most searches for goods and services seek nearby results; achieving this requires network-based spatial constraints or at least as-the-crow-flies distance rankings for the search. A WGRS geocode, like US.DC.WAS.55, could easily be used to focus searches geographically, especially given a Web-mapping interface. Gazetteers based on associations of sub-grid-scale names-Capitol Hill, Foggy Bottom, the Mall-with WGRS equivalents also can be imagined, such as US.DC.WAS.CapitolHill.

At this time, WGRS is a proposal for a community-licensed, public standard following the examples of the Internet and the Web. For WGRS to be workable, the georeferencing mechanisms and notations outlined in this paper need to be reviewed, perhaps revised, and eventually ratified by a consortium of academic, business, and government interests. A single enterprise designated by the consortium must conduct the standards "exercise" of defining and maintaining the regional and local grids on a worldwide basis, with assistance by local experts as necessary. The resulting database of grids and associated software to access it also must be published in numerous venues-hardcopy and digital media and, of course, the wired and wireless Webs-for convenient access by all.

Implementation of WGRS thus amounts to the creation and maintenance of a globally accessible gazetteer, not only of the WGRS grids, but also public and private place names, abbreviations, populations (and undoubtedly much other spatial data and information). This is a very large database undertaking, which also must be accomplished pro bono, probably in the private sector. The currency, privacy, and security of the data will certainly be issues; equality and equity of access to the data also will need to be considered.

\section{Conclusion}

This paper has outlined the conceptual background and technical specifications of a new World Geographic Reference System. This system offers a computer- and user-friendly mechanism for specifying location, both in the real world and in facsimiles of it, i.e., in GPS, GIS, LBS and Web applications generally. The fundamental unit of the system is a set of culturally anchored mapping 
grids, which fit well with human spatial cognition. In contrast to mapping frameworks such as MGRS and UTM, which tile space arbitrarily, WGRS ties grids to centers of human activity, precisely where the need for mapping is greatest. The WGRS references are always areal, at some scale, explicitly recognizing the approximation inherent in georeferencing.

We see WGRS as a vital link between the theoretical and the practical aspects of georeferencing for the Internet age. We have used the title "A New World Geographic Reference System" deliberately, since there have been many other such grid system proposals, even one that has used the same name (cf. ASCE/ACSM/ASPRS 1994). Like a single map projection, a single grid system cannot solve all cartographic problems, of course. Nevertheless, if ease of use is chosen first among the factors cited by Goodchild (1994), then WGRS may facilitate a bridge between basic geography, location-based services the World Wide Web. Specifically, we look forward to a day when LBS appliances and Web pages embed explicitly spatial WGRS references directly in HTML (or similar) textual code, for example: $<$ a gref=wgrp://US.DC.WAS.54.18.28.83.11 $>$ The Washington Monument $</ a>$. With such a geographical extension, many new and exciting personal mobility and Web applications will become possible.

\section{ACKNOWLEDGMENTS}

S. Lee Hancock, a co-inventor on all the WGRSrelated patents, contributed to this paper in numerous ways. The authors also thank Val Noronha of the University of California Santa Barbara and three anonymous reviewers.

\section{REFERENCES}

ASCE/ACSM/ASPRS. 1994. Glossary of the mapping sciences. ACSM/ASPRS, Bethesda, Maryland.

Clarke, K.C. 1995. Analytical and computer cartography. Englewood Cliffs, New Jersey: Prentice Hall.

Clarke, K.C. 2002. Criteria and measures for the comparison of global geocoding systems. In: M.F. Goodchild and A.J. Kimerling (eds), Discrete Global Grids, chapter 8. [Web Book at http:// www.ncgia.ucsb.edu/globalgrids-book/].

Crampton, J.W. 1998. The convergence of spatial technologies. Cartographic Perspectives 30 (Spring). [http://www.nacis.org/cp/cp30/cp_essay.html].

Dana, P.H., and S.L. Hancock. 1997. A user friendly location reference system. In: Proceedings of the National Technical Meeting, Santa Monica, California. The Institute of Navigation, Washington, D.C.

Dutton, G.H. 1997. A hierarchical coordinate system for geoprocessing and cartography: Working through the scales. Ph.D. Dissertation, University of Zurich. Heidelberg, Germany: Springer-Verlag.

Goodchild, M.F. 1994. Criteria for evaluation of global grid models for environmental monitoring and analysis. Handout from NCGIA Initiative 15. See Spatial Analysis on the Sphere: A Review, by Rob Raskin, NCGIA Technical Report 94-7.

Goodchild, M.F., and J. Proctor. 1997. Scale in a digital geographic world. Geographical and Environmental Modeling 1(1): 5-23.

Hancock, S.L., and P.H. Dana. 1998. U.S. Patent 5,839,088, issued November 17, 1998: "Geographic Location Referencing System and Method;" further developed in U.S. Patents 6,047,236, 6,202,023, 6,339,744, 6,356,834 issued April 4, 2000, March 13, 2001, January 15, 2002, and March 12, 2002, respectively.

Kimerling, A.J., K. Sah, D. White, and L. Song. 1999. Comparing geometrical properties of global grids. Cartography and Geographic Information Systems 26(4): 271-88.

NCGIA. 2001. Specialist meeting on location-based services. Center for Spatially Integrated Social Science, December 14-15, 2001. [http://csiss.ncgia.ucsb.edu/ events/meetings/location-based/].

Raper, J.F., D.W. Rhind, and J.W. Shepherd. 1992. Postcodes: The new geography. New York, New York: Longman Scientific and Technical.

Rosenberg, D.K. 2000. The open source software licensing page.[http://www.stromian.com/Open_Source_ Licensing.htm].

Snyder, J.P. 1987. Map projections-A working manual. U.S. Geological Survey Professional Paper 1395. Washington, D.C.: U.S. Government Printing Office.

Tobler, W.R., and Z. Chen. 1986. A quadtree for global information storage. Geographical Analysis 18(4): 360-71.

World Wide Web Consortium. 2002. Naming and addressing: URIs, URLs, ... [http://www.w3.org/ Addressing/; last revised 2002/07/09]. 